\title{
méthode pratique de calcul d'un pieu isolé à l'aide du pénétromètre statique
}

par

\section{G. Philipponnat}

Chef de la Division Géotechnique du C.E.B.T.P.

Conférence prononcée devant le Comité Français de Mécanique des Sols le 19 mars 1979

\section{Introduction}

La littérature relative au calcul des pieux est particulièrement vaste. La lecture des diverses publications ou des traités de mécanique des sols permet de réunir une multitude d'éléments fort intéressants sur le comportement des pieux ainsi que sur le pénétromètre statique. Mais en fin de compte bien des points restent mal définis ce qui conduit parfois à des divergences très importantes tant au niveau des notes de calcul que des comparaisons avec les essais de chargement.

Alors qu'il existe des méthodes pratiques simples et relativement précises pour déterminer par exemple la contrainte admissible sur une semelle à partir des caractéristiques C et $\varphi$ ou les contraintes admissibles sur les semelles et sur les pieux à partir des essais pressiométriques. Les bureaux de contrôle ont constaté qu'une méthode pratique de calcul d'un pieu isolé basée sur l'essai de pénétration statique restait à mettre au point.

Dans le cadre d'une action de concertation technique permanente de ces bureaux, une méthode a été élaborée par un groupe de travail qui comprenait outre l'auteur de cet exposé :

- Messieurs Bergin et Isnard de Veritas

- Monsieur Boucherie de Socotec

- Monsieur Soria du C.E.P.

ainsi que Messieurs D. Gouvenot de Solétanche et J. Hurtado de Sols et Structures qui ont bien voulu s'associer à notre travail et nous apporter une aide précieuse.

Des règles pratiques pour l'interprétation des essais de pénétration statique en vue du calcul des pieux sont utilisées depuis longtemps et il n'est pas possible de rappeler ici l'ensemble de ces travaux : nous nous limiterons à citer les noms de Begemann, de Beers, Dinesh Mohan, I'Herminier, Sanglerat, Van der Veen.

Dans le dossier Fond 72 , les Laboratoires des Ponts et Chaussées ont présenté en se basant sur les travaux des auteurs qui viennent d'être cités, des recommandations pour le calcul des pieux. C'est ce texte qui a été retenu comme base de départ de nos travaux.
Les buts que nous avons poursuivis ont été au nombre de trois :

- mettre au point une méthode simple et d'application aussi précise que possible de façon à réduire le facteur personnel :

- tenir compte du type de pieu et de son mode d'exécution pour évaluer le frottement latéral,

- étalonner la méthode et ajuster au mieux les coefficients numériques entrant en jeu.

Nous allons successivement décrire cette méthode, puis commenter les étalonnages qui ont pu être faits à partir de 44 essais de chargement de pieux.

\section{Exposé de la méthode}

\subsection{Cas étudiés}

\section{Nombre de couches - définitions}

- Cas d'un sol homogène - Fig. 1 a

- Cas d'un multicouche -- Fig. 1 b

- Il s'agit réellement d'un multicouche uniquement si la contrainte verticale effective $\sigma_{v}^{\prime}$ due aux couches supérieures est au moins égale à $1.10^{5} \mathrm{~Pa}$.

$-\mathrm{H}$ est la fiche totale du pieu à ne pas confondre avec $D$ - D est l'ancrage dans la couche porteuse

\subsection{Formules générales et notations}

Charge nominale (charge admissible vis-à-vis de la résistance du sol)

Formule générale : $Q_{N}=\frac{A}{F_{s 1}} a_{p}+\frac{Q_{1}}{F_{s 2}}$

avec :

$A=$ aire de la section droite du pieu

$\mathrm{q}_{\mathrm{p}}=$ résistance de pointe à la rupture

$\mathrm{Q}_{\mathrm{f}}=$ frottement latéral mobilisable le long du fût à la rupture

$F_{s 1}=$ coefficient de sécurité sur le terme de pointe

$\mathrm{F}_{\mathrm{s} 2}=$ coefficient de sécurité sur le terme de frottement latéral 

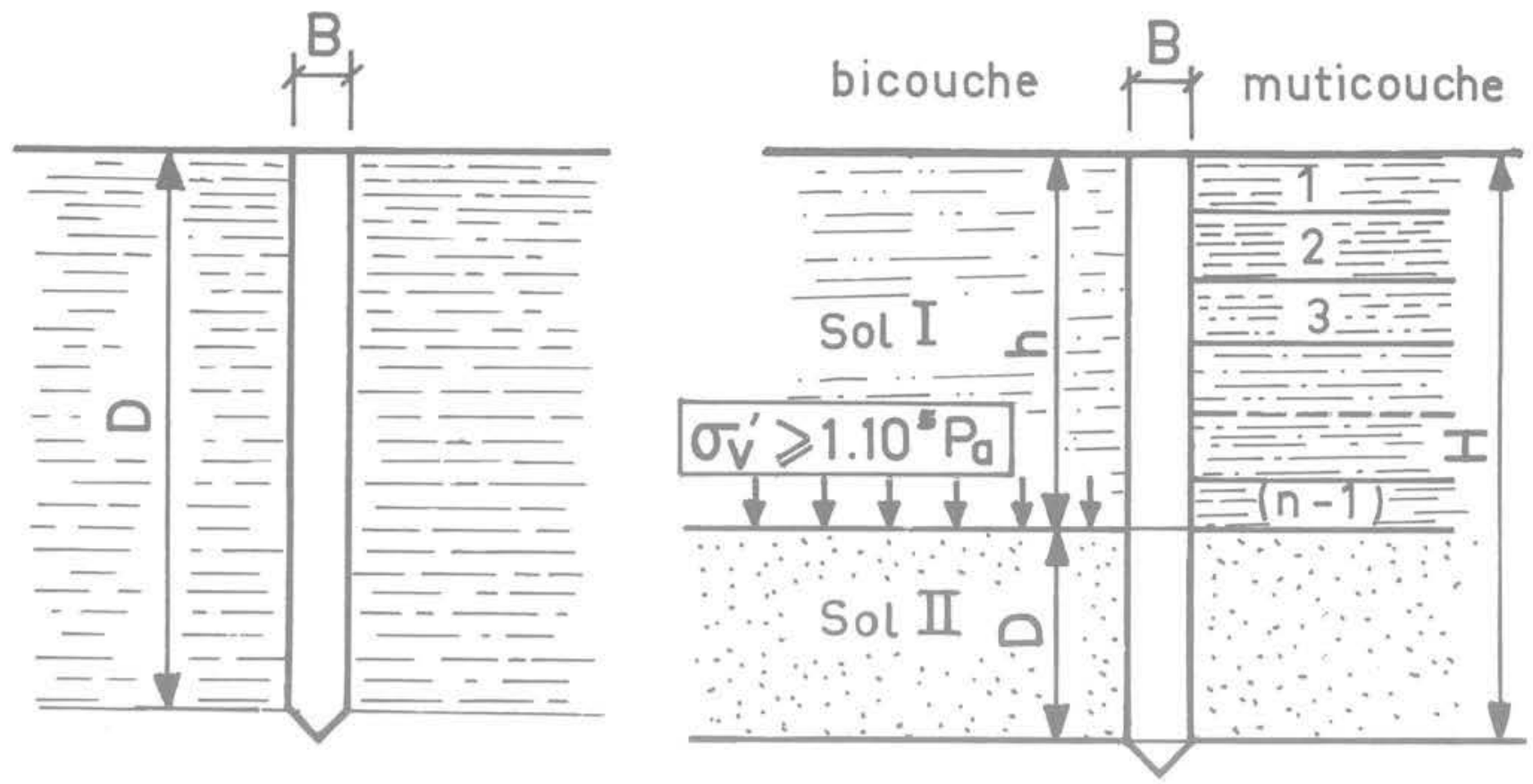

Cas d'un sol homogène

\section{Cas d un multicouche}

Fig. 1 Définition du sol: sol homogène et multicouches

Valeur des coefficients de sécurité

En général, les coefficients de sécurité adoptés sont:

$$
\begin{aligned}
& \text { Résistance de pointe : } \mathrm{F}_{\mathrm{s} 1}=3 \\
& \text { Frottement latéral: } \quad \dot{\mathrm{F}}_{\mathrm{s} 2}=2
\end{aligned}
$$

Ces coefficients ne sont pas homogènes entre eux ni avec le coefficient de sécurité pris pour interpréter un essai de chargement (1). Le dépouillement que nous avons fait d'un certain nombre d'essais de chargement montre que les formules actuelles permettant de calculer ia résistance limite de pointe des pieux à partir du pénétromètre statique conduisent à des résultats trop optimistes.

Ces formules ont donc été réajustées en tenant compte de cette remarque :

avec les formules que nous proposons et uniquement avec celles-ci on pourra adopter: $\mathrm{F}_{\mathrm{s} 1}=\mathrm{F}_{\mathrm{s} 2}=2$

Contrainte admissible (vis-à-vis de la charge nominale)

$$
\mathrm{q}_{\mathrm{a}}=\frac{\mathrm{Q}_{\mathrm{N}}}{\mathrm{A}}
$$

Frottement latéral global

avec :

$$
\mathrm{Q}_{\mathrm{f}}=\mathrm{p} \sum \mathrm{f}_{\mathrm{ui}} \mathrm{h}_{\mathrm{i}}
$$

$$
\begin{aligned}
& \mathrm{p}=\text { périmètre du pieu } \\
& \mathrm{f}_{\mathrm{ui}}=\begin{array}{l}
\text { frottement unitaire mobilisable dans la } \\
\text { couche } \mathrm{i}
\end{array} \\
& \mathrm{h}_{\mathrm{i}}=\text { épaisseur de la couche } \mathrm{i}
\end{aligned}
$$

(1) En général, la charge nominale est déduite de la charge de rupture obtenue au cours d'un essai de pieu en appliquant un coefficient de sécurité de deux. L'interprétation basée sur la valeur de la charge de fluage qui a tendance a se développer actuellement n'a pu être testée faute de données suffisantes.

\subsection{Détermination de la résistance limite du sol $q_{1}$}

\section{Formule générale}

Pour une couche d'ancrage donnée, on écrira :

$$
q_{1}=a_{p} q_{c}
$$

avec :

$\mathrm{q}_{\mathrm{c}}=$ résistance unitaire de pointe au pénétromètre statique dans la couche d'ancrage

Le coefficient $\alpha_{p}$ relie la résistance de pointe limite du pieu à la résistance au pénétromètre statique. Les valeurs de $\alpha_{p}$ que nous donnons dans le tableau $\mathrm{A}$ ont été établies par comparaison avec des essais de chargement réels.

Remarques:-Ces valeurs sont sensiblement plus faibles que celles données par d'autres auteurs, cependant, le coefficient de sécurité que nous préconisons $\mathrm{F}_{\mathrm{s} 1}$ étant de 2

au lieu de 3 , les contraintes admissibles en pointe ne sont que peu modifiées.

- Les différences de résistances de pointe entre pieu et pénétromètre ont souvent été attribuées à un « effet d'échelle \%. En fait, les résultats ne font pas apparaître d'écart entre pieux de diamètres très différents, comme d'ailleurs l'avaient déjà montré les essais de Saint-RémyLès-Chevreuses. En fait, la différence réside bien entre pieux et pénétromètres et pourrait être due à la vitesse d'enfoncement. Cette vitesse est de $2 \mathrm{~cm} / \mathrm{s}$ pour les pénétromètres et sera par exemple de $10 \mathrm{~cm}$ en $24 \mathrm{~h}$ pour un pieu d'essai, soit de $10^{-4} \mathrm{~cm} / \mathrm{s}$. Les caractéristiques mécaniques des sols, comme on le sait, dépendent fortement de la vitesse d'application des charges. En ce qui concerne, le cas particulier des graves, l'effet d'échelle vient probablement se superposer à ce phénomène. 


\begin{tabular}{l|l}
\multicolumn{1}{c|}{ Nature du sol } & $\alpha_{\mathrm{p}}$ \\
\hline Argile et craie & 0,50 \\
Limon & 0,45 \\
Sable & 0,40 \\
Grave & 0,35 \\
\hline \multirow{2}{*}{ Tableau A } & VALEURS de $\alpha_{p}$
\end{tabular}

- Détermination de $q_{f}$ dans un sol hétérogène (fig. 2)

Lorsque le sol est hétérogène, la résistance de pointe d'un pieu de gros diamètre B est influencée par une certaine hauteur de sol "a" au-dessus de la pointe et "c " en dessous.

Les variations de résistances enregistrées par le pénétromètre sont « laminées \#.

On remplacera alors $q_{c}$ par $q_{c}^{\prime}$ dans la formule (4).

La valeur de $q_{c}^{\prime}$ est déterminée en prenant la moyenne arithmétique des résistances de pointe au pénétropètre sur les hauteurs " a " et " $c$ ».

Soit : $\mathrm{q}_{\mathrm{ca}}$ la résistance moyenne sur la hauteur " $\mathrm{a}$ »

$\mathrm{q}_{\mathrm{cc}}$ la résistance moyenne sur la hauteur « $\mathrm{C}$ »

on pose :

$$
\mathrm{q}_{\mathrm{c}}^{\prime}=\frac{\mathrm{q}_{\mathrm{ca}}+\mathrm{q}_{\mathrm{cc}}}{2}
$$

Cette méthode est due à Van der Veen.

Les valeurs attribuées aux longueurs « a » et « $\mathrm{c}$ » varient selon les auteurs. Van der Veen préconise d'adopter $\mathrm{a}=\mathrm{B}$, $\mathrm{c}=3,75 \mathrm{~B}$. Pour notre part, nous avons pris $\mathrm{a}=\mathrm{c}=3 \mathrm{~B}$.

Cependant, lorsque les valeurs de $q_{f}$ sont très dispersées, it faut procéder à un examen critíque de la résistance obtenue par la formule 5. En particulier, les" pointes trop marquées du diagramme de pénétration doivent être éliminées, de même si $q_{c a}>q_{c c^{\prime}}$ il est prudent de prendre $\mathrm{q}_{\mathrm{ca}}=\mathrm{q}_{\mathrm{cc}}$

\section{Garde minimum sous la pointe des pieux:}

Lorsque la couche d'ancrage repose sur une couche molle, il convient d'assurer une garde minimum de quatre diamètres sans toutefois descendre en dessous de 3 mètres par rapport à une couche molle sous-jacente, ceci, en dehors de toute considération sur l'effet des groupes de pieux.

Dans certains cas particuliers (micropieux par exemple), cette règle pourra être transgressée mais moyennant une reconnaissance détailiée pour s'assurer de l'épaisseur de la couche porteuse.

\subsection{Détermination de la profondeur d'ancrage critique $\mathrm{D}_{\mathrm{c}}$}

Etant donné la différence d'échelle entre les diamètres des pénétromètres statiques et des pieux courants, il est difficile de passer de l'un à l'autre pour déterminer la profondeur d'ancrage critique.

La méthode proposée est basée sur les critères suivants : Condition 1 : La profondeur minimale d'ancrage que nous appellerons $D_{\min }$ est donnée par la plus grande des valeurs suivantes:

- monocouche : $3,00 \mathrm{~m}$ ou 6 diamètres

- multicouche : $1,50 \mathrm{~m}$ ou 3 diamètres

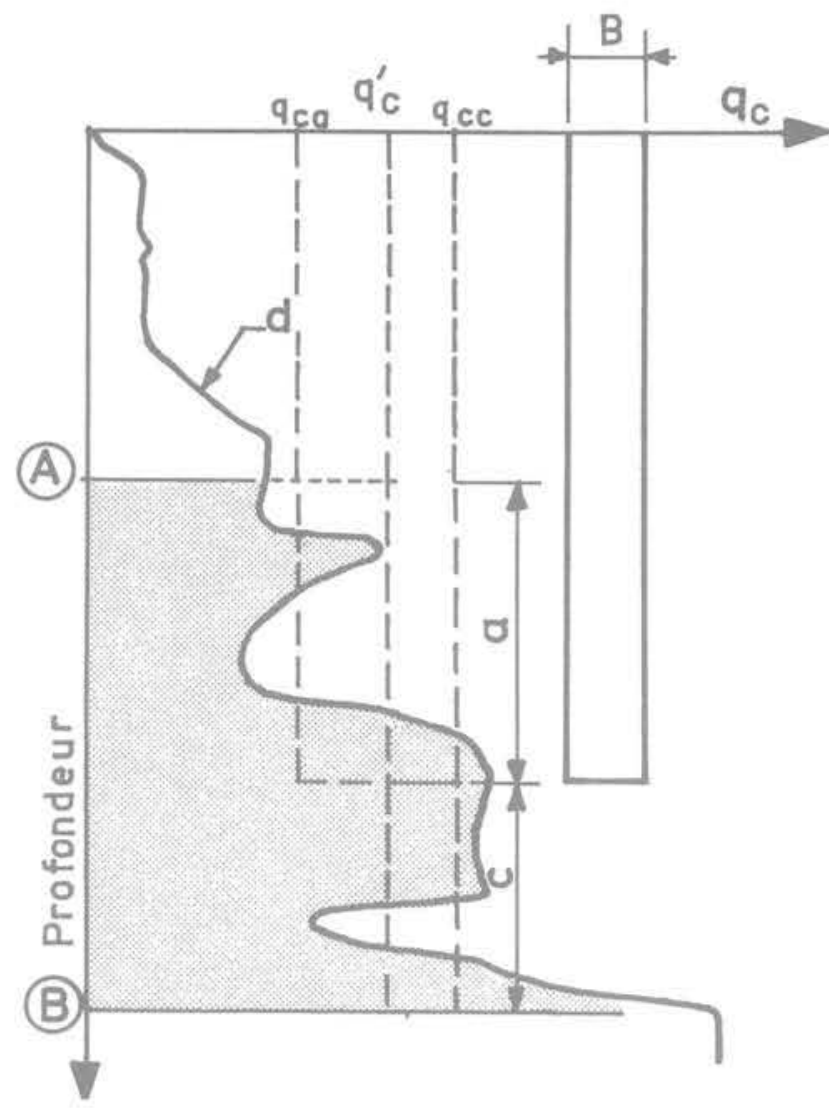

Fig. 2 Pondération de la résistance de pointe dans un sol hétérogène

En effet, dans le cas d'un monocouche, si les conditions ci-dessus ne sont pas respectées, il ne s'agit plus d'une fondation profonde mais superficielle ou semi-profonde.

Dans le cas d'un multicouche, cette condition est souhaitable pour tenir compte de l'imprécision qui règne sur les limites entre couches et des écarts possibles entre les cotes réelles d'arrêt des pieux et les cotes théoriques. Cette règle n'est évidemment pas valable pour les pieux s'appuyant sur un rocher franc.

Condition 2: La profondeur d'ancrage nécessaire pour mobiliser $q_{\text {f }}$ est donnée par la formule de Foray et Puech pour les sols à angle de frottement interne élevé. Pourles sols à comportement cohérent, la condition 1 est suffisante.

Cette formule s'écrit :

$D_{c}=25 \sqrt{B}\left(1+\frac{q_{l}}{10}\right)$

avec $q_{\text {, en }} \mathrm{MPa}$, $B$ et $\mathrm{D}_{\mathrm{c}}$ en $\mathrm{cm}$

Dans la pratique, on pourra adopter comme profondeur d'ancrage critique:

- $D_{\min }$ pour les sols argileux (condition 1)

- Pour les sols sableux, la profondeur donnée par l'abaque de la fig. 4 qui tient compte des conditions précédentes.

A partir de la résistance de pointe limite $q_{l}$ et du diamètre $B$, on lit directement en ordonnée sur l'abaque la valeur de la profondeur d'ancrage $D_{c}$ (échelle de gauche: milieu homogène, échelle de droite: multicouche).

Remarques:- Cet abaque n'est valable que pour les diamètres courants $(B \leqslant 180 \mathrm{~cm})$.

- Dans le cas d'un multicouche tel que les couches supérieures conduisent à une contrainte effective $\sigma_{v}^{\circ}<10^{5} \mathrm{~Pa}$ 


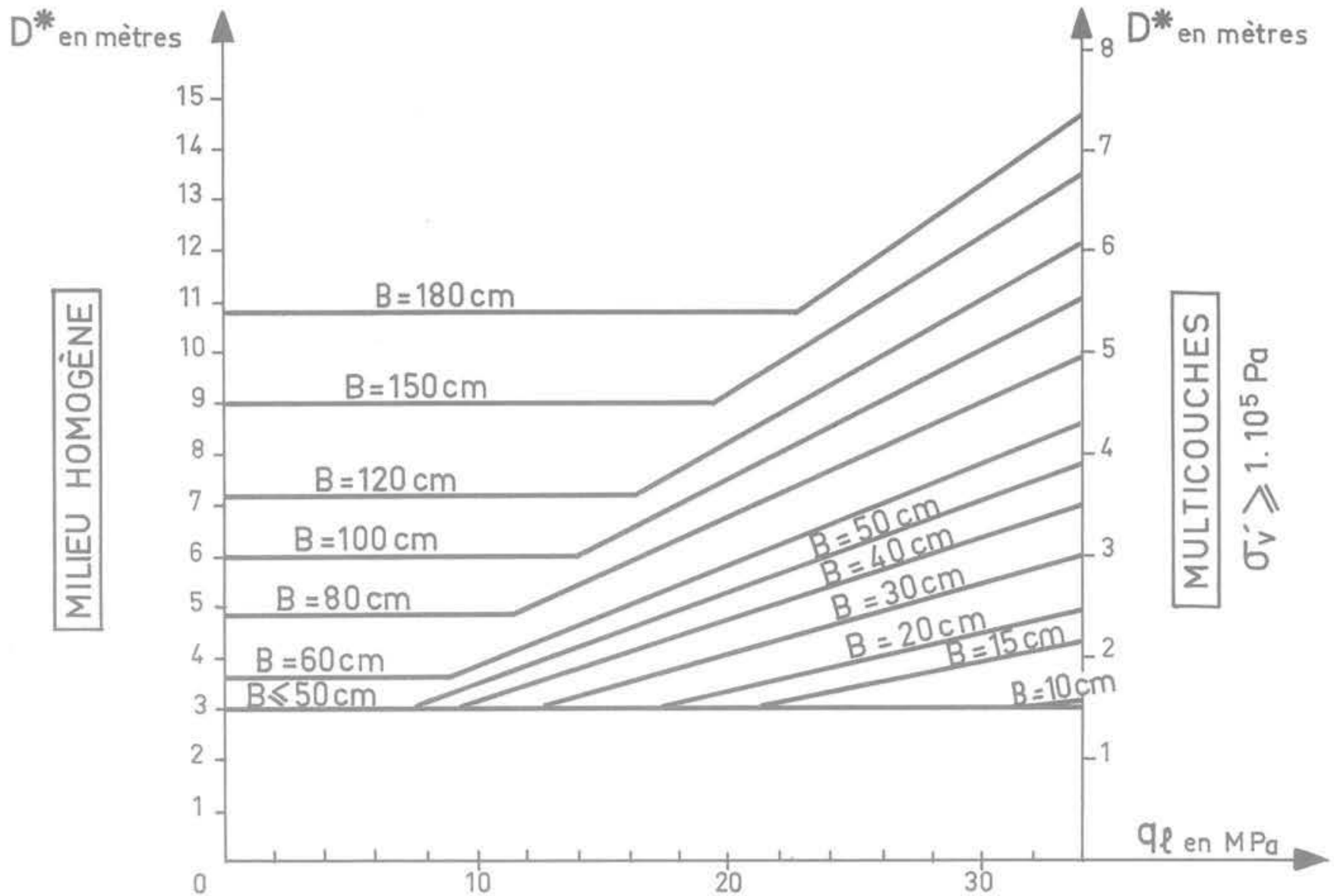

Fig. 3 Détermination de la profondeur d'ancrage pour les sols à angle de frottement interne élevé

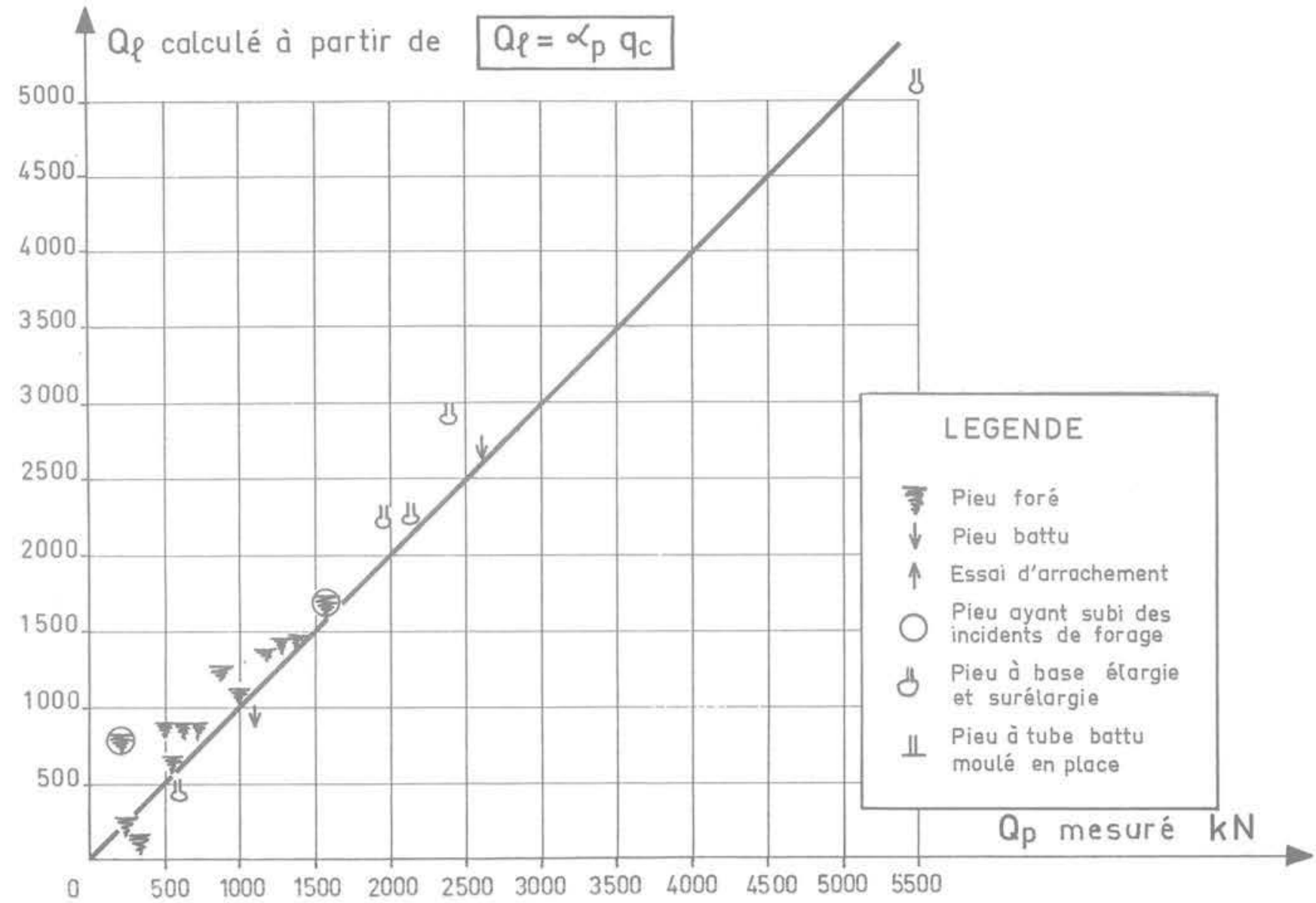

Fig. 4 Comparaison des charges de rupture en pointe 
au niveau du toit de la couche d'ancrage, on déterminera l'ancrage critique par une interpolation proportionnelle à $\sigma_{v}^{\prime}$ entre le cas du sol homogène et celui du bicouche.

- Compte tenu des contraintes intrinsèques données par le D.T.U et donc des contraintes limites de pointe correspondantes, on constate que pour la plupart des cas courants, l'ancrage $D_{\min }$ est suffisant. Ce résultat rejoint a posteriori la règle habituelle des trois diamètres d'ancrage pour le multicouche.

\subsection{Détermination du frottement latéral $Q_{\text {, }}$}

II faut déterminer $Q_{\text {f }}$ pour l'introduire dans la formule (1) mais en fait $Q$,est calculé à partir de la formule (3) et c'est en définitive le frottement latéral unitaire $f_{u}$ mobilisable dans chaque couche au contact sol-pieu qu'il faut apprécier.

II existe plusieurs façons d'évaluer le frottement latéra d'un pieu à partir du pénétromètre statique. Les pénétromètres statiques sont de plus en plus souvent munis d'un manchon situé à proximité immédiate de la pointe. Ce dispositif fait actuellement l'objet de Recommandations aux niveaux européen et international.

D'autre part, grâce a l'interprétation statistique de dizaines de milliers d'essais, G. Sanglerat et l'Ecole Centrale Lyonnaise ont pu établir des corrélations assez précises entre le frottement latéral unitaire et la résistance de pointe du pénétromètre statique. Dans ces corrélations, la nature du sol intervient.

II en découle que l'on peut relier le frottement latéral unitaire entre le pieu et le sol $f_{u}$ à la résistance de pointe $q_{c}$ du pénétromètre statique à condition de tenir compte:

$$
\text { - de la nature du sol }
$$$$
\text { - du type de pieu }
$$

ce que nous traduirons par la formule suivante :

avec :

$$
f_{u}=\alpha_{i} \frac{q_{c}}{\alpha_{s}}
$$

$\alpha_{s}$ : coefficient fonction du sol

$\alpha_{f}^{s}$ : coefficient fonction du pieu

Les valeurs de $\alpha_{\mathrm{s}}$ et de $\alpha_{t}$ à prendre en compte pour les calculs sont données dans les tableaux $\mathrm{B}$ et $\mathrm{C}$ ci-après:

\begin{tabular}{l|c}
\multicolumn{1}{c|}{ Nature du sol } & $\alpha_{s}$ \\
\hline Argile et craie & 50 \\
$\begin{array}{l}\text { Silt, argile sableuse } \\
\text { Sable argileux } \\
\text { Sable lâche }\end{array}$ & 60 \\
Sable moyen & 100 \\
Sable dense & 150 \\
Graves & 200 \\
\hline
\end{tabular}

\section{Tableau B}

Remarques: - Les valeurs de $\alpha$ correspondent pratiquement à celles préconisées par d'áutres auteurs pour l'évaluation du frottement latéral sur les pieux.
- II ne faut pas confondre $f_{u}$ avec $f_{\text {s }}$ qui est le frottement latéral mesuré sur manchon dans une zone où le sol est peu perturbé et où il peut $y$ avoir une influence des contraintes transmises par la pointe.

- Les valeurs de $\alpha_{f}$ figurant dans le tableau C ont été obtenues à partir de celles données par d'autres auteurs et surtout du dépouillement d'essais de chargement.

- Pour les sables moyens de résistance de pointe $5<\mathrm{q}_{\mathrm{c}}<20 \mathrm{MPa}$, on pourra adopter la formule :

$$
\alpha_{\mathrm{s}}=45 \mathrm{q}_{\mathrm{c}}^{\frac{1}{2}} \text { avec } \mathrm{q}_{\mathrm{c}} \text { en } \mathrm{MPa}
$$

- Compte tenu des incertitudes qui règnent actuellement sur la valeur réelle du frottement mobilisable et de la dispersion observée dans les résultats d'essais sur pieux réels instrumentés, il apparaît indispensable de limiter supérieurement la valeur du frottement latéral $f_{u}$.

Les valeurs maximales de $f_{u}$ à prendre en compte figurent dans le tableau C. Ces valeurs ne pourront être dépassées qu'en se basant sur une expérience locale réelle et en particulier sur les résultats d'essais de chargement.

C'est ainsi que dans certaines craies ou marnes, on admet des frottements latéraux sensiblement supérieurs. De même, l'injection sous haute-pression de coulis peut améliorer le frottement latéral dans des proportions très importantes.

\section{Comparaison avec des essais de chargement}

\subsection{Description des essais}

Afin de pouvoir comparer valablement la charge nominale calculée à partir de la méthode qui vient d'être exposée à celle obtenue réellement, il fallait réunir des comptes rendus d'essais de chargement pour lesquels un certain nombre de renseignements étaient connus, à savoir au minimum :

- le type du pieu testé, ses caractéristiques géométriques son mode de mise en place,

- la courbe de chargement/enfoncement du pieu jusqu'à ce que la rupture soit au moins amorcée,

- le diagramme de pénétration statique au droit du pieu, - la nature des couches traversées.

Lorsque les pieux étaient instrumentés, la répartition entre l'effort de pointe et le frottement latéral au moment de la rupture était connue. Dans quelques autres cas, les pieux ont fait l'objet d'un double chemisage total et la résistance du pieu à la rupture était alors égale à celle de la pointe. La plupart des essais qui ont été pris en compte ont fait l'objet de publications tant en France qu'à l'étranger. Au total, l'étalonnage a pu être faít sur 44 pieux présentant les caractéristiques suivantes :

$$
\text { - Nombre total de pieux : } 44
$$

$$
\text { - Nombre de sites différents : } 15
$$

Nature du sol d'ancrage:

$\begin{array}{ll}\text { - grave } & : 1 \text { pieu } \\ \text { - sable compact } & : 9 \text { pieux } \\ \text { - sable moyen } & : 3 \text { pieux } \\ \text { - sable lâche } & : 2 \text { pieux } \\ \text { - limon } & : 7 \text { pieux dont } \\ & \\ \text { - argile des flandres } & : 7 \text { pieux } \\ \text { - argile raide fissurée } & : 12 \text { pieux } \\ \text { - craie altérée } & : 3 \text { pieux }\end{array}$

limon sableux : 3 limon argileux : 4 ou argile sableuse

Diamètre des pieux : répartition

$\begin{array}{llllll}\text { Diamètre en } \mathrm{cm}(<50) & 50 & 75 & 100 & 125 & 155\end{array}$

$\begin{array}{llllll}\text { Nombre de pieux } & 12 & 17 & 7 & 0 & 8\end{array}$ 


\begin{tabular}{|c|c|c|c|}
\hline $\begin{array}{l}\text { Nature du pieu } \\
\text { au contact } \\
\text { du sol }\end{array}$ & Type du pieu & $\alpha_{i}$ & $\begin{array}{c}\text { Valeur } \\
\text { maximale de } \\
f_{u} \text { en kpa }\end{array}$ \\
\hline \multirow{3}{*}{ Béton } & $\begin{array}{l}\text { - Pieux façonnés à l'avance } \\
\text { - Pieux à tube battu exécutés en place (1) } \\
\text { - Pieux forés à tubage vibro-foncé } \\
\text { (vibration du béton) } \\
\text { - Pieux injectés }\end{array}$ & 1,25 & 120 \\
\hline & $\begin{array}{l}\text { - Pieux forés de diamètre }<1,50 \mathrm{~m} \\
\text { - Pieux Benoto }\end{array}$ & 0,85 & $\begin{array}{l}100 \\
120 \text { dans } \\
\text { la craie }\end{array}$ \\
\hline & $\begin{array}{l}\text { - Pieux forés de gros diamétres }>1,50 \mathrm{~m} \\
\text { - Barrettes moulées } \\
\text { - Pieux foncés } \\
\text { - Puits coulés à pleine fouille }\end{array}$ & 0,75 & 80 \\
\hline \multirow{3}{*}{ Métallique } & - Pieux H (sur le périmètre circonscrit) & 1,10 & 120 \\
\hline & $\begin{array}{l}\text { - Palpieux, pieux métalliques battus } \\
\text { - Pieux foncés }\end{array}$ & 0,60 & 50 \\
\hline & $\begin{array}{l}\text { - Pieux forés à chemisage perdu, ex: } \\
\text { pieux métalliques ouverts mis en } \\
\text { œuuvre à la Benoto }\end{array}$ & 0,30 & 25 \\
\hline $\begin{array}{l}\text { Béton ou } \\
\text { métallique }\end{array}$ & - Pieux lancés à l'eau ou à la bentonite & \multicolumn{2}{|c|}{$\begin{array}{l}\text { négliger le frottement } \\
\text { latéral sur la partie } \\
\text { lancée }\end{array}$} \\
\hline
\end{tabular}

(1) Pieux à tube récupéré uniquement

Valeur de $\alpha_{\text {, }}$

Tableau C

Longueur des pieux : $6,70 \mathrm{~m}$ à $32,50 \mathrm{~m}$

Type de pieux :

- Pieux façonnés à l'avance (pieux battus) : :6

- Pieux battus moulés : 8

- Pieux Franki à base élargie $\quad: 5$

- Pieux Franki à base surélargie : :9

- Pieu injecté $\quad: 1$

- Pieux forés à la tarière simple : 8

- Pieux forés à la boue $\quad: 4$

- Pieux Benoto $\quad: 2$

- Pieu foré à la tarière continue : : 1

Les chiffres entre parenthèses indiquent le nombre de pieux métalliques
3.2 Etude de la répartition entre pointe et latéral (Pieux intrumentés)

Dans 15 cas, les pieux étaient instrumentés et il était alors possible de connaitre l'effort de pointe et le frottement latéral au moment de la rupture. Dans d'autres cas, pieux Franki (à base surélargie), les pieux avaient fait l'objet d'un double chemisage sur la totalité, la résistance réelle de la pointe du pieu est alors mesurée directement.

\section{Charge limite en pointe}

Connaissant la charge limite réelle de rupture en pointe $q_{1}$. il est alors possible de calculer la valeur réelle correspondante du coefficient $\alpha_{p}$ entrant dans la formule (4) 


\begin{tabular}{|c|c|c|c|c|c|}
\hline \multirow{2}{*}{$\begin{array}{l}\text { Nature } \\
\text { du sol(1) }\end{array}$} & \multirow{2}{*}{$\begin{array}{l}\text { Nombre de } \\
\text { résultats }\end{array}$} & \multirow{2}{*}{ Diamètre } & \multirow{2}{*}{$\begin{array}{l}\text { Type } \\
\text { de pieu }\end{array}$} & \multicolumn{2}{|c|}{ Valeurs réelles de $\alpha_{p}$} \\
\hline & & & & moyennes & extrêmes \\
\hline Argile & 10 & 0,5 à $1,40 \mathrm{~m}$ & $\begin{array}{l}\text { Tarière } \\
\text { simple } \\
\text { Franki }\end{array}$ & 0,43 & 0,3 à 0,64 \\
\hline Limon & 4 & $0,56 / 1,37 \mathrm{~m}$ & $\begin{array}{l}\text { Tarière boue } \\
\text { Benoto } \\
\text { Franki }\end{array}$ & 0,43 & 0,38 à 0,54 \\
\hline Sable & 3 & $35 \times 35 / 1,53$ & $\begin{array}{l}\text { Pieu battu } \\
\text { Franki }\end{array}$ & 0,37 & $0,315 / 0,43$ \\
\hline Grave & 1 & 0,60 & Métal battu & 0,335 & - \\
\hline
\end{tabular}

Enfin le seul essai réalisé dans la grave est d'interprétation douteuse car le diagramme pénétrométrique ne dépasse pas la pointe du pieu.

En dehors de ces cas le tableau de la page précédente mérite deux remarques:

- Tout d'abord, les valeurs moyennes semblent bien décroître lorsque l'on passe de l'argile à la grave, mais cette diminution n'est pas très significative. $M$. Cassan a proposé pour sa part d'adopter une valeur unique de $\alpha$. Toutefois, le nombre d'essais actuellement en notre possession est insuffisant pour proposer une meilleure approximation de la valeur de la résistance de pointe du pieu.

- Ensuite, et c'est là le fait marquant, les résultats obtenus sur 18 essais montrent d'une façon indiscutable que le rapport $\alpha$ entre résistance de pointe des pieux et des pénétromêtres est de 0,3 à 0,5 et non de 0,7 à 1 comme il est habituellement admis. C'est là un résultat nouveau très marquant obtenu par le groupe de travail, évidemment sur la base des données disponibles.

\section{Frottement latéral}

Le frottement latéral dépendant de deux paramètres $\alpha_{\text {f }}$ et

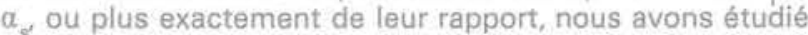
les valeurs réelles de $\alpha_{\mathrm{f}}$ en adoptant pour $\alpha_{\mathrm{s}}$ les valeurs théoriques. Rappelons que $\alpha$ est un coefficient qui dépend du sol et non du type de contact sol-pieu et que l'influence de la nature du sol a fait l'objet d'une étude statistique très complète de G. Sanglerat par comparaison de la résistance de pointe et du frottement sur manchon.

Par contre, le coefficient $\alpha$, qui dépend de la nature du pieu mérite un examen très attentif.

Les résultats obtenus peuvent être rassemblés dans le tableau suivant:

\begin{tabular}{|c|c|c|c|c|c|c|c|}
\hline \multirow{2}{*}{$\begin{array}{l}\text { Nature } \\
\text { du fût }\end{array}$} & \multirow[t]{2}{*}{ Type de pieu } & \multirow[t]{2}{*}{ Nature du sol } & \multirow[t]{2}{*}{ Diamètre } & \multirow{2}{*}{$\begin{array}{l}\text { Nombre } \\
\text { de mesure }\end{array}$} & \multicolumn{2}{|c|}{$\alpha_{f}$} & \multirow{2}{*}{$\begin{array}{c}\alpha_{f} \\
\text { selon } \\
\text { méthode } \\
\text { de calcul }\end{array}$} \\
\hline & & & & & médiane & extrêmes & \\
\hline \multirow{5}{*}{ Béton } & Pieu injecté & silt sableux & $30 \times 30$ & 1 & 1,23 & & 1,25 \\
\hline & $\begin{array}{l}\text { Pieu façonné à } \\
\text { l'avance }\end{array}$ & sable compact & $35 \times 35$ & 1 & 1,25 & & 1,25 \\
\hline & Tarière simple & argile & $0,70 / 1 \mathrm{~m}$ & 7 & 0,88 & $0,72 / 1,36$ & \\
\hline & Tarière à la boue & $\begin{array}{l}\text { craie } \\
\text { limon argileux } \\
\text { sable compact }\end{array}$ & $\begin{array}{c}0,56 \mathrm{a} \\
0,9\end{array}$ & 4 & 1,24 & $1,07 / 1,49$ & 0,85 \\
\hline & Benoto & limon argileux & 0,9 & 1 & 0,62 & - & \\
\hline \multirow{2}{*}{ Métal } & $\begin{array}{l}\text { Façonné à } \\
\text { l'avance }\end{array}$ & grave & 0,64 & 1 & 0,75 & - & 0,6 \\
\hline & $\begin{array}{l}\text { Tube métallique } \\
\text { façonné à la } \\
\text { Benoto }\end{array}$ & limon argileux & 0,88 & 1 & \multicolumn{2}{|c|}{$\begin{array}{l}0,22 \text { a } 0,39 \\
\text { en fonction du temps } \\
\text { de repos }\end{array}$} & 0,30 \\
\hline
\end{tabular}

(1) Un essai dans la craie qui donnait une valeur aberrante a été áliminé (faute probable d'exécution). D'autre part, un essai effectué dans une argile sableuse de faible consistance a donné une valeur de $a_{\text {ple }}$ lordre de 0,7 à 1 (diagramme de pénétration peu précis). Cet essai n'a pas été pris en compte. 
Trois observations sont à faire :

- Le nombre de pieux instrumentés est trop faible pour en tirer des conclusions définitives.

- Quoi qu'il en soit, les coefficients déduits des essais de pieux sont en assez bon accord avec ceux retenus. On observe cependant que les 4 pieux instrumentés réalisés à la tarière sous boue donnent des frottements latéraux élevés.

- L'examen détaillé des essais relatifs aux pieux forés confirme comme l'a montré par ailleurs D. Gouvenot que le frottement latéral peut varier considérablement (du simple au double) selon la qualité de l'exécution du pieu.

Comparaison des valeurs calculées aux valeurs mesurées

Les figures 4 et 5 présentent sous une autre forme l'exploitation qui a pu être faite des pieux instrumentés, ces figures font ressortir :

- la bonne concordance d'ensemble tant pour la pointe que pour le frottement latéral

- la dispersion des résultats en latéral

- le fait que malgré les valeurs adoptées pour le coefficient $\alpha_{p}$, les valeurs calculées en pointe sont encore légèrement plus élevées que les valeurs réelles.

3.3 Comparaison globale de la méthode de calcul et des essais de chargement

\section{Evaluation des charges limites}

Si le nombre de pieux instrumentés dont nous disposions était limité, il a été par contre possible de se faire une idée globale de la validité de la méthode proposée en comparant pour l'ensemble des pieux la charge limite calculée et la charge limite réelle. Cette comparaison est représentée sur la figure 6 .

Cette figure montre entre autres qu'en appliquant un coefficient de sécurité de 2 comme préconisé sur la charge limite théorique, le coefficient de sécurité réel varie selon les essais de chargement étudiés entre 1,6 et 2,7 à l'exception de deux essais pour lesquels la méthode conduit à une évaluation pessimiste.

Au-delà de la dispersion inhérente d'une part aux aléas d'exécution des pieux mais aussi d'autre part, au fait qu'il existe parfois une hétérogénéité non négligeable des terrains, (dans certains cas des essais de pénétration exécutés à proximité immédiate les uns et des autres présentent des écarts notables), la méthode de calcul retenue fournit une évaluation acceptable de la charge limite. Lorsque les résultats de calcul sont optimistes, les différences sont modérées et ne mettent pas en jeu la sécurité de l'ouvrage. De même les écarts dans l'autre sens sont généralement modérés, seuls quelques cas en nombre limité, fournissent des valeurs réelles sensiblement plus élevées que ne le laisse prévoir le calcul.

Si l'on tient compte de la grande disparité des principaux paramètres :

- type de pieux

- diamètre des pieux

- nature des sols traversés

l'estimation de la charge limite des pieux à partir de la méthode préconisée peut être considérée comme très satisfaisante.

\section{Tassement des pieux sous la charge nominale}

Nous avons relevé sur les courbes de chargement/ enfoncement des pieux, le tassement correspondant à la charge nominale déduite de la méthode de calcul.

On obtient la répartition suivante :

\begin{tabular}{|c|c|c|c|c|}
\hline Tassements s & \multicolumn{2}{|c|}{$\begin{array}{c}\text { Pieux battus } \\
\text { ou à tubes battus }\end{array}$} & \multicolumn{2}{|c|}{ Pieux forés } \\
\hline sous $a_{N}$ & Nombre & $\%$ & Nombre & $\%$ \\
\hline $\mathrm{s}<5 \mathrm{~mm}$ & 19 & 73 & 9 & 60 \\
\hline $5<s<10 \mathrm{~mm}$ & 6 & 23 & 4 & 27 \\
\hline $10<\mathrm{s}<20 \mathrm{~mm}$ & 1 & 4 & 2 & 13 \\
\hline \multicolumn{2}{|c|}{$\mathrm{s}>20 \mathrm{~mm}$} & & \multicolumn{2}{|c|}{ néant } \\
\hline
\end{tabular}

\section{Conclusion}

L'objectif que s'était fixé la commission de concertation des bureaux de contrôle a été atteint en présentant cette méthode de calcul de la charge limite des pieux basée sur l'essai de pénétration statique. Comme on a pu le constater, cette méthode est simple et donne des estimations réalistes du comportement des pieux.

D'autre part, le facteur personnel est réduit au maximum, ce qui éliminera les risques de divergences importants entre les utilisateurs.

Par contre, nous sommes tout à fait conscient que le nombre de pieux instrumentés analysé est assez faible et que les valeurs des différents coefficients numériques qui ont été proposées sont susceptibles d'être modifiées dans l'avenir en fonction des expérimentations futures de façon à mieux cerner la réalité. De ce point de vue, la multiplication des essais de chargement de pieux instrumentés est infiniment souhaitable.

Enfin, comme il l'a déjà été mentionné, le frottement latéral est influencé très fortement par la qualité de l'exécution en particulier pour les pieux forés. Les valeurs numériques proposées pour l'estimation de frottement latéral correspondent aux pratiques d'exécution courantes actuelles.

Si, comme c'est déjà le cas avec des pieux injectés sous forte pression, les techniques d'exécution progressent de façon à garantir une meilleure mobilisation du frottement latéral, il sera très certainement possible d'augmenter les valeurs numériques du coefficient $\alpha_{f}$ entrant dans le calcul et donc la charge admissible-sur le pieu. 

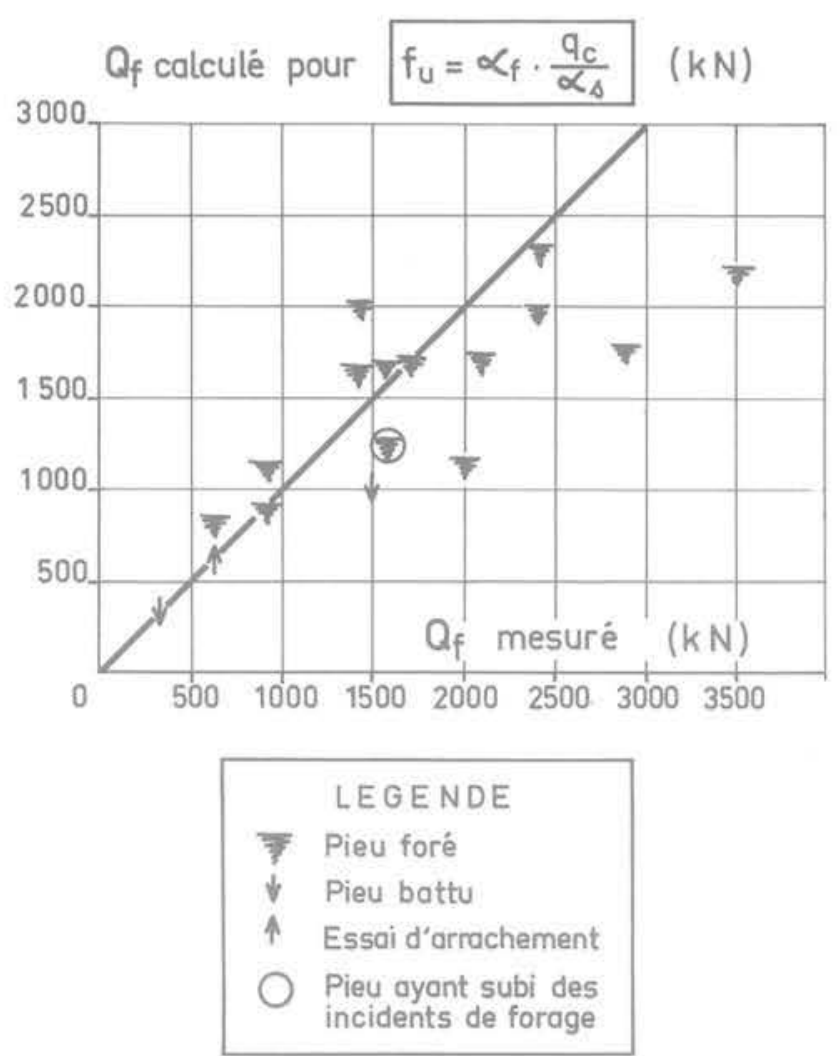

Fig. 5 Comparaison du frottement latéral total

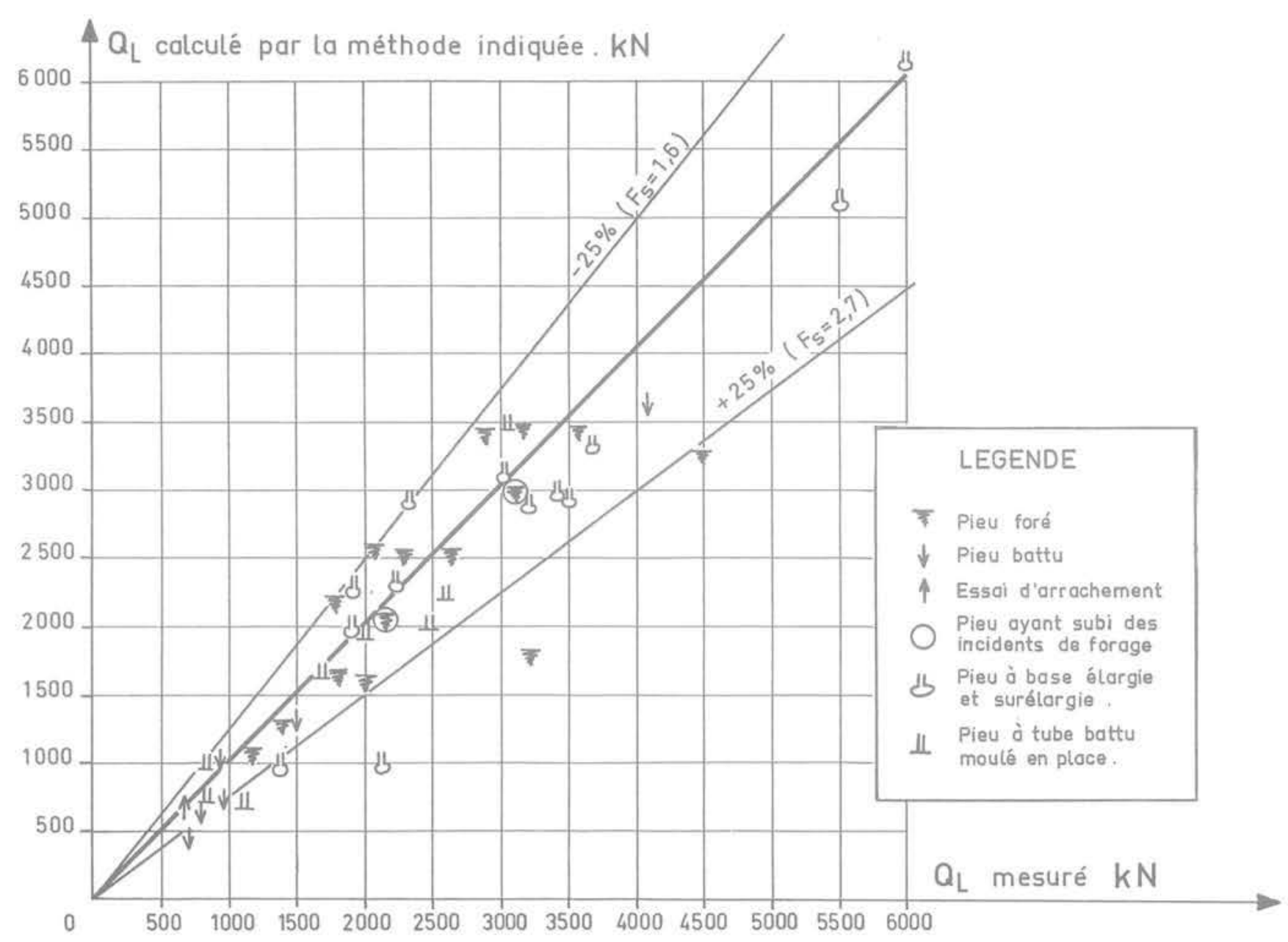

Fig. 6 Comparaison des charges limites globales 


\section{Références Bibliographiques}

1 - E. DE BEER - "Méthodes de déduction de la capacité portante d'un pieu à partir des résu/tats des essais de pénétration Annales des T.P. de Belgique $n^{\circ}$ 4-5-6 - 1971/1972.

2 - E. DE BEER et Al - «Bearing capacity of displacement piles in stiff fissured clays $\#-$ Comptes rendus de recherches I.RSIA $n^{\circ} 39$ - Mars 1977.

3 - B.B. BROMS - «Methods of calculating the ultimate bearing capacity of piles \#-A summary - Revue Sols-Soils $n^{\circ} 18$ $19-1966$

4-M. BUSTAMANTE et M. PANET - ir Essai statique d'arrachement d'un pieu - M.V-Trindel N - Bulletin L.C.P.C. $n^{\circ} 79-$ 1975.

5 - M. BUSTAMANTE, M. DERVAUX et $Y$. HULO - "Essais de chargement statique de trois pieux lancés battus »-Bulletin L.C.P.C $n^{\circ} 84-1976$.

6 - M. BUSTAMANTE et D. GOUVENOT - « Incidence des conditions d'exécution et du délai de repos sur le comportement et la portance des pieux forés 》 - Conférence du 9 janvier 1978 au C.F.M.S.

7 - O. COMBARIEU - "Essais de chargement de pieux forés dans un limon argileux \# - Bulletin L.C.P.C. $n^{\circ} 80$ - 1975.

8-0. COMBARIEU - "Essais de chargement de pieux de grande longueur battus dans la grave et de l'argile raide „ - Bulletin L.C.P.C. $n^{\circ} 82-1976$.
9 - DINESH MOHAN and VIRENDRA KUMAR - $"$ Load bearing capacity of piles 》 - Géotechnique. The international Journal of Soil Mechanics - volume XIII - march $1963 n^{\circ} 1$ p. 76-86.

10 - FOND 72 - "Fondations courantes d'ouvrages d'art " Document L.C.P.C. - S.E.T.R.A. - 1972.

11 - J. FLORENTIN et Al - «Observations faites sur la craie comme couche de fondation \#-Compte rendu du Congrès International de Mécanique des sols Paris 19

12 - D. GOUVENOT - "Essais en France et à l'étranger sur le frottement latéral en fondation: amélioration par injection » Revue travaux - Novembre 1973

13 - M. WALLAYS - "Pieux Franki à bases surélargies » Annales I.T.B.T.P. $n^{\circ} 300$ - Décembre 1972.

14-G. SANGLERAT - "The penetrometer and soil exploration 》 - Elsevier - Amsterdam - 1972.

15 - G. SANGLERAT - "Le pénétromètre statique-dynamique et ses diverses applications pratiques 》-Conférence - 1977.

16 - G. SANGLERAT et AI - "Classification directe des sols a l'aide du pénétromètre statique avec manchon de mesure du frottement latéral » - Annales I.T.B.T.P. $n^{\circ} 340-1976$.

17 - A. VESIC - «Design of pile fondations \# - National cooperative Highway Research Programm Synthesis of highway practice $n^{\circ} 42$. Transportation research Board, National Research Council Washington D.C. 1977. 